\title{
Analysis of pulmonary nodules caused by nontuberculous mycobacteriosis in 101 resected cases: multi-center retrospective study
}

\author{
Naoko Ose ${ }^{1 \wedge}$, Yukiyasu Takeuchi ${ }^{2}$, Naoto Kitahara ${ }^{3}$, Akihide Matumura ${ }^{4}$, Ken Kodama ${ }^{5}$, \\ Hiroyuki Shiono $^{6}$, Yoshiyuki Susaki ${ }^{7}$, Yasunobu Funakoshi ${ }^{8}$, Hiroyuki Takabatake ${ }^{9}$, Yasushi Shintani ${ }^{1}$; \\ for the Thoracic Surgery Study Group of Osaka University (TSSGO)
}

${ }^{1}$ Osaka University Graduate School of Medicine, Department of General Thoracic Surgery, Suita-shi, Osaka, Japan; ${ }^{2}$ Osaka Toneyama Medical Center, Department of General Thoracic Surgery, Toyonaka-shi, Osaka, Japan; ${ }^{3}$ Osaka Habikino Medical Center, Department of General Thoracic Surgery, Habikiho-shi, Osaka, Japan; ${ }^{4}$ Kinki-chuo Chest Medical Center, Department of General Thoracic Surgery, Sakai-shi, Osaka, Japan; ${ }^{5}$ Yao Municipal Hospital, Department of General Thoracic Surgery, Yao-shi, Osaka, Japan; ${ }^{6}$ Kindai University Nara Hospital, Department of General Thoracic Surgery, Ikoma-shi, Nara, Japan; ${ }^{7}$ Osaka International Cancer Institute, Department of General Thoracic Surgery, Chuo-ku, Osaka-shi, Osaka, Japan; ${ }^{8}$ Osaka General Medical Center, Department of General Thoracic Surgery, Sumiyoshi-ku, Osaka-shi, Osaka, Japan; ${ }^{9}$ Suita Municipal Hospital, Department of General Thoracic Surgery, Suita-shi, Osaka, Japan

Contributions: (I) Conception and design: N Ose; (II) Administrative support: Y Takeuchi, Y Shintani; (III) Provision of study materials or patients: All authors; (IV) Collection and assembly of data: N Ose; (V) Data analysis and interpretation: N Ose, Y Shintani; (VI) Manuscript writing: All authors; (VII) Final approval of manuscript: All authors.

Correspondence to: Naoko Ose, MD. Department of General Thoracic Surgery, Osaka University Graduate School of Medicine, 2-2(L5) Yamadaoka, Suita-shi, Osaka 565-0871, Japan. Email: naokoose@thoracic.med.osaka-u.ac.jp.

Background: Solitary pulmonary nodules caused by nontuberculous mycobacteriosis are included as a category of pulmonary nontuberculous mycobacterium disease. Clinical characteristics, treatments and prognosis are not fully known because there are a few related reports.

Methods: This was a multi-center retrospective study of 101 cases diagnosed as solitary nodular type of nontuberculous mycobacteriosis from January 2000 to March 2017 that underwent resection at 9 related facilities belonging to the Thoracic Surgery Study Group of Osaka.

Results: The most common pathogen was Mycobacterium avium complex $(\mathrm{n}=77,87.5 \%$ ), followed by Mycobacterium kansasii ( $\mathrm{n}=8,9.1 \%$ ). Chest computed tomography results showed subpleural locations that were difficult to distinguish from lung cancer. Fluorodeoxyglucose positron emission tomography/computed tomography was performed in 58 cases and positive results were obtained in 35 (60.3\%), with an average maximum standardized uptake value of 3.87. The purpose of resection in most cases was for diagnosis. The surgical procedure was wedge resection in 87 , segmentectomy in 3 , and lobectomy in 11 , while 77 underwent thoracoscopic surgery. Postoperative complications occurred in 7 cases, though no infections caused by nontuberculous mycobacteriosis were noted. The median observation period was 27 months. A worsened condition occurred in 10 (9.9\%) with Mycobacterium avium complex, though none had local recurrence.

Conclusions: Solitary pulmonary nodules due to nontuberculous mycobacteriosis is difficult to diagnose based on preoperative examination results or distinguish from lung cancer. Among the present cases, none had local complications or recurrence, even in those that underwent a wedge resection, thus postoperative chemotherapy was not considered necessary if a complete resection was performed. On the other hand, some cases showed reinfection after a long period following resection, thus patients should be informed of that future possibility.

$\wedge$ ORCID: 0000-0002-3509-9536. 
Keywords: Solitary; pulmonary; nodule; nontuberculous mycobacterial disease (NTM)

Submitted Oct 19, 2020. Accepted for publication Dec 18, 2020.

doi: $10.21037 /$ jtd-20-3108

View this article at: http://dx.doi.org/10.21037/jtd-20-3108

\section{Introduction}

Pulmonary nontuberculous mycobacterial disease (NTM) is classified into 5 categories; fibrocavitary (FC) type, featuring a cavity and scattered shadows in the upper lung field similar to pulmonary tuberculosis, nodular/ bronchiectatic (NB) type, which is characterized by thickening and dilation of the bronchial wall with small multiple nodules in the middle and lingular segment, and solitary nodular (SN-NTM) type, showing isolated nodules in the lung field, as well as systemic seeding type and hot tub lung type. The latter two are not local disease, thus neither is indicated for surgical treatment, whereas the FC and NB types may benefit from surgical treatment as part of multidisciplinary therapy. Although preand postoperative chemotherapy are necessary and surgical treatment has only an auxiliary role, adaptation of surgical treatment for $\mathrm{FC}$ or NB type is based on guidelines published in 2007 by the American Society of Thoracic Surgery (ATS) and the American Society of Infectious Diseases (1), as well as the Guidelines of the British Society of Thoracic Surgery (BTS), revised in 2017 (2). On the other hand, no treatment method for SN-NTM type, which is often caused by Mycobacterium avium complex (MAC), is described in those guidelines. It has been pointed out that some differences in the immune system may cause SN-NTM (3), though not NTM pulmonary disease, and asymptomatic cases that need to be distinguished from lung cancer are often encountered. The ATS guidelines show that SN-NTM type caused only by MAC does not require postoperative chemotherapy if complete resection is performed (Grade C, level III) (1). And the BTS guidelines says that following resection of a solitary NTM nodule in an individual with no other features of NTM-pulmonary disease, antibiotic treatment is not usually required (Grade D) (2).

However, the guidelines do not provide descriptions for species other than MAC and there are few related reports, thus clinical characteristics and effective treatments for cases affected by other organisms have not been clarified. In addition, consideration must be given regarding whether anatomical resection is necessary, the same as with FC/NBNTM (4). The present retrospective study was performed to clarify clinical background findings, treatment contents, and prognosis of SN-NTM type patients based on multicenter experiences.

We present the following article in accordance with STROBE reporting checklist (available at http://dx.doi. org/10.21037/jtd-20-3108).

\section{Methods}

This retrospective study complied with STROBE guidelines (5). This study included 101 patients who were diagnosed SN-NTM. The clinical relevance was evaluated based on the clinical background, chemotherapy, surgical procedure, prognosis, and other important factors from medical records.

\section{Patients}

A total of 101 resected cases diagnosed as SN-NTM type from January 2000 to March 2017 at 9 related facilities belonging to the Thoracic Surgery Study Group of Osaka were enrolled (Figure 1). Only NTM cases identified by culture or mycobacterial genetic testing were targeted regardless of pre- or postoperative diagnosis. The patients' characteristics are shown in Table 1.

\section{Definition of SN-NTM}

SN-NTM type was defined when NTM was isolated from resected tissue. At least two doctors including radiologist and respiratory surgeon regarded a lesion as nodule not FC or NB type on computed tomography (CT) image. Definitions used for identification testing and imaging are noted following.

\section{NTM identification}

All cases underwent mycobacterial smearing and acid-fast bacterium culture testing of resected tissue. Fluorescent staining was used to confirm the presence of acid-fast bacterium. Mycobacterial culturing was done using Ogawa 


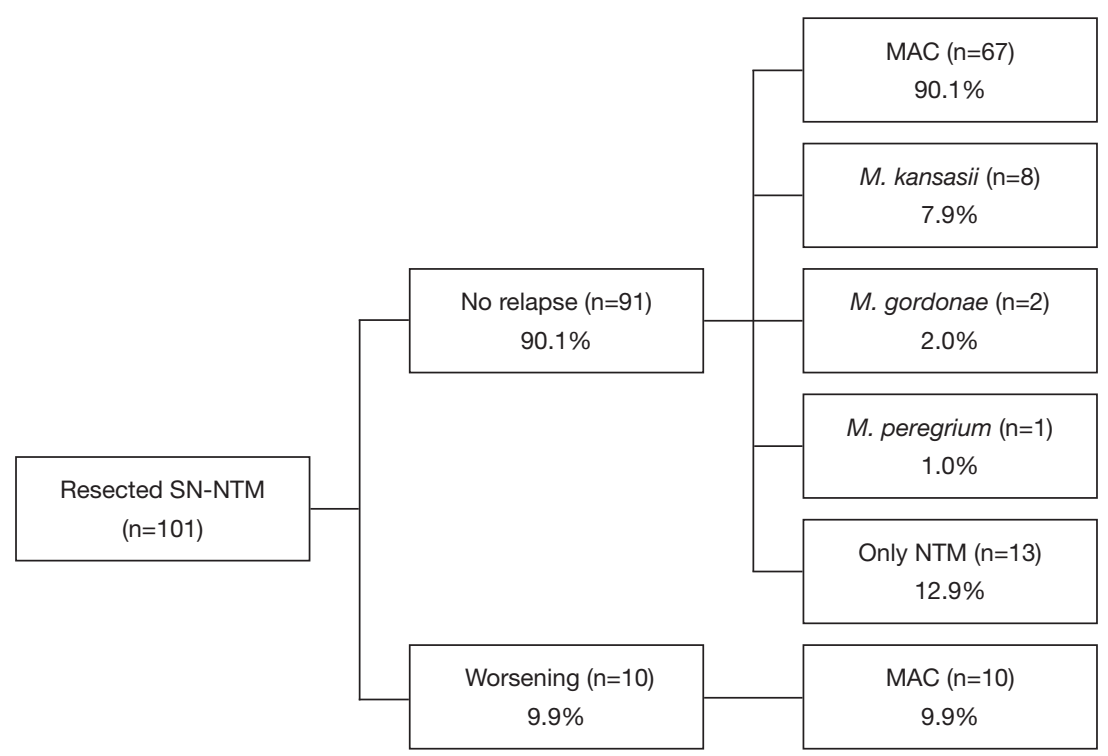

Figure 1 Study flow for enrolment of this study describing the numbers of relapse and worsening patients with pathogens.

medium or liquid medium included with the Mycobacteria Growth Indicator Tube system. Identification of Mycobacterium (M) tuberculosis, M. avium, or M. intracellulare was done using a polymerase chain reaction assay with a nucleic acid amplification protocol (PCR), transcriptionreverse transcription concerted reaction method using an rRNA detection reagent (TRC), or loop-mediated isothermal amplification method, and varied according to the facility and was not necessarily enforced in all cases. Since a species identification test was not performed by some facilities, those cases were classified as "NTM (unclassified)". Cases in which NTM was not isolated were excluded, even if pathological examination results indicated an epithelial granuloma or were positive in Ziehl-Neelsen staining, including those that could not be distinguished from an $M$. tuberculosis infection.

\section{Diagnostic imaging}

At each of the participating institutions, two or more physicians, including radiologists, respiratory physicians, and/or surgeons, judged each solitary pulmonary nodule using chest CT results, including some nodules that showed a slight spray-type shading around them. Number and mass size were not considered. A nodule was defined as adjacent when part was in contact with the pleura and as subpleural when located in one-third of the outer lung field. The nodule including air inside which was not considered as cavity were included. In cases that underwent a fluoro-18 fluorodeoxyglucose positron emission tomography/ computed tomography (FDG-PET) examination, significant accumulation was defined as a maximum standardized uptake value (SUVmax) of 2.5 or greater, when that description was included.

\section{Prognosis}

Follow-up period and type of examination performed were dependent on each individual facility and the attending physicians. Worsening was defined when bacteriological relapse occurred after surgery or postoperative chemotherapy treatment, or when the appearance or spread of NTM infection was suspected in chest CT findings, which included reinfection and recurrence cases.

\section{Statistical analysis}

Variables are expressed as the mean \pm standard deviation. Comparisons between 2 groups were made using a MannWhitney $\mathrm{U}$ test, with a chi-square test used for categorical variables. All analyses were performed using the JMP 15.1.0 statistical software package (SAS Institute Inc., USA).

\section{Ethics approval}

The study was conducted in accordance with the 
Table 1 Patient characteristics

\begin{tabular}{|c|c|}
\hline & Cases $(n=101)$ \\
\hline Sex (male/female) & $59 / 42$ \\
\hline Age & $61.8 \pm 11.4[29-80]$ \\
\hline \multicolumn{2}{|l|}{ Symptom } \\
\hline None & 92 \\
\hline Positive & 9 \\
\hline Cough & 5 \\
\hline Hemosputum & 2 \\
\hline Sputum & 1 \\
\hline Chest pain & 1 \\
\hline Fatigue & 1 \\
\hline Numbers (single/multiple) & $88 / 13$ \\
\hline \multicolumn{2}{|l|}{ Locations } \\
\hline Right/left & $72 / 29$ \\
\hline Upper & $41 * / 10$ \\
\hline Middle/lingular & $9 * / 4$ \\
\hline Lower & $23 / 15$ \\
\hline \multicolumn{2}{|l|}{ Pathogen } \\
\hline MAC & 77 \\
\hline M. kansasii & 8 \\
\hline M. gordonae & 2 \\
\hline M. peregrinum & 1 \\
\hline Only NTM & 13 \\
\hline \multicolumn{2}{|l|}{ Diagnosis } \\
\hline Preoperative & 7 \\
\hline Bronchoscopy & 2 \\
\hline CTGB & 1 \\
\hline Gastric juice culture & 1 \\
\hline No details & 3 \\
\hline Postoperative & 94 \\
\hline
\end{tabular}

*, one case had two nodules in right upper and middle lobe. MAC, mycobacterium avium complex, M., mycobacterium; NTM, nontuberculous mycobacteriosis; CTGB, computed tomography guided biopsy.

Declaration of Helsinki (as revised in 2013). This study was initially approved by the Institutional Review Board of Osaka University Hospital (project approval No. 17270) in December 2017 and thereafter by each of the participating
Table 2 Results of imaging examination

\begin{tabular}{lc}
\hline & Cases $(\mathrm{n}=101)$ \\
\hline Chest CT findings & $19.1 \pm 8.2[7-59]$ \\
Size $(\mathrm{mm})$ & $100(99.0 \%)$ \\
Subpleural & $60(59.4 \%)$ \\
Adjacent pleura & $36(35.6 \%)$ \\
Pleural indentation & $30(29.7 \%)$ \\
Spiculation & $14(13.9 \%)$ \\
Satellite lesion & $13(12.9 \%)$ \\
Necrosis & $7(6.9 \%)$ \\
Air inside & $3(3.0 \%)$ \\
Calcification & \\
FDG-PET & 43 \\
None & $58(2.5-13.2)$ \\
Done & $23(0-2.3)$ \\
SUVmax $\geq 2.5$ & 58 \\
SUVmax $<2.5$ &
\end{tabular}

CT, computed tomography; FDG-PET, fluorodeoxyglucoseposition emission tomography.

institutions. Individual consent for this retrospective analysis was waived.

\section{Results}

Patient background information is shown in Table 1. Nine cases $(8.9 \%)$ were symptomatic. Localization of the nodules was left side in 29 (upper division 10, lingular 4, lower 15) and right side in 72 (upper 41, middle 9, lower 23). The average mass diameter was $19.1 \pm 8.2 \mathrm{~mm}(7-59 \mathrm{~mm})$. Preoperative diagnosis was obtained in only 7 cases, based on results of bronchoscopic biopsy in 2, CT guided biopsy in 1 , and gastric juice culture in 1, though a bronchoscopic examination was performed in only 27 patients (positive rate $7.4 \%$ ). Mycobacterial examination was PCR-positive in 39, TRC-positive in 4, culture-positive in 55 . The most common pathogen was MAC (n=77, 87.5\%), followed by $M$. kansasii $(\mathrm{n}=8,9.1 \%)$.

Imaging results are summarized in Table 2. Chest CT results of 100 cases showed subpleural locations, which were difficult to distinguish from lung cancer because of indentation and spiculation in many. FDG-PET was performed in 58 cases suspected to be lung cancer and 
Table 3 Treatment and prognosis

\begin{tabular}{|c|c|c|}
\hline & \multicolumn{2}{|c|}{ Cases $(n=101)$} \\
\hline & No. & $\begin{array}{l}\text { Treatment } \\
\text { period }\end{array}$ \\
\hline \multicolumn{3}{|l|}{ Operation } \\
\hline Wedge & 87 & \\
\hline Segmentectomy & 3 & \\
\hline Lobectomy & 11 & \\
\hline Approach (thoracotomy/VATS) & $24 / 77$ & \\
\hline Operating time (min) & $112(37-360)$ & \\
\hline Blood loss (g) & $5(0-1,240)$ & \\
\hline Drainage period (day) & $3(1-21)$ & \\
\hline Complications & 7 & \\
\hline Late onset alveolopleural fistula & 3 & \\
\hline Chylothorax & 2 & \\
\hline Pneumonia & 1 & \\
\hline Prolonged air leakage & 1 & \\
\hline Preoperative chemotherapy & 7 & \\
\hline $\mathrm{H}+\mathrm{R}+\mathrm{E}$ & 3 & 1, 3, 9 months \\
\hline $\mathrm{H}$ & 1 & 1 months \\
\hline $\mathrm{R}+\mathrm{E}+\mathrm{C}+\mathrm{LVFX}$ & 1 & 3 months \\
\hline $\mathrm{C}+\mathrm{E}$ & 1 & 0.5 months \\
\hline$A Z M+R+E$ & 1 & 8 months \\
\hline Postoperative chemotherapy & 21 & \\
\hline $\mathrm{R}+\mathrm{E}+\mathrm{C}$ & 5 & $\begin{array}{c}6,8,14, \\
29 \text { months }\end{array}$ \\
\hline $\mathrm{H}+\mathrm{R}+\mathrm{E}$ & 4 & 1,9 months \\
\hline C & 3 & \\
\hline Spafroxasin & 2 & \\
\hline $\mathrm{R}+\mathrm{E}+\mathrm{C}+\mathrm{LVFX}$ & 1 & 6 months \\
\hline R+LVFX & 1 & \\
\hline LVFX & 1 & 2 months \\
\hline C+LVFX & 1 & 12 months \\
\hline$A Z M+R+E$ & 1 & 12 months \\
\hline $\mathrm{C}+\mathrm{E}$ & 1 & 0.5 months \\
\hline $\mathrm{H}+\mathrm{R}+\mathrm{E}+\mathrm{Z}$ & 1 & \\
\hline \multicolumn{3}{|l|}{ Reinfection } \\
\hline Yes & 10 & \\
\hline Sputum culture positive & 5 & \\
\hline Nodules with other lobe & 5 & \\
\hline Nodules with same lobe & 3 & \\
\hline No & 91 & \\
\hline
\end{tabular}

VATS, video assisted thoracic surgery; $H$, isoniazid; $\mathrm{R}$, rifampicin; E, ethambutol; C, clarithromycin; LVFX, levofroxasin; AZM, azithromycin; Z, pyrazinamide. ${ }^{\ddagger}$, treatment period was described only in cases it was certain. positive results were obtained in $35(60.3 \%)$, with an average SUVmax of 3.87 .

Treatment and prognosis findings are presented in Table 3. The purpose of resection was for diagnosis in most cases. In cases diagnosed as NTM infection, surgery was selected because of suspected combined lung cancer or based on patient wish to stop chemotherapy. The surgical procedure was a wedge resection in 87 , segmentectomy in 3 , and lobectomy in 11 , while 77 underwent thoracoscopic surgery. Postoperative complications occurred in 7 cases, though no infections were caused by NTM. Preoperative chemotherapy was performed in 7 cases, for suspicion of $M$. tuberculosis in 4 cases with a regimen of 3 drugs (isoniazid, rifampicin, ethambutol) and in 3 cases diagnosed with NTM for therapeutic purposes. Postoperative chemotherapy was performed in 21 cases.

The median observation period was 27 months. A worsened condition was found in 10 cases, including relapse within 1 year after resection in 2 cases and reinfection with MAC more than 1 year later in 8 (9.9\%) (Table 4). Only 2 cases showed worsening soon after surgery, though neither was local recurrence, as 1 demonstrated a worsened shadow in the contralateral lobe and bacterial relapse occurred one month after surgery (Case 1), while other had infection in a wedge resected lobe and other lobes on the contralateral side at 6 months after the operation shown by CT results (Case 8). Eight cases considered to be reinfection had that occur at 1.5 to 5 years after surgery (average 3.2 years) and only 1 had a lesion in the same lobe, but it was difficult to distinguish between reinfection and recurrence. Of 21 patients who underwent postoperative chemotherapy, 4 had a worsened condition. No recurrence of $M$. kansasii was seen in 7 of the 8 cases without postoperative chemotherapy. No deaths from NTM infection were noted. There was no significant difference of clinical features between no relapse and worsening cases (Table 5).

\section{Discussion}

Only 6 studies describing treatment of SN-NTM type in more than 10 cases have been published $(3,6-10)$, including 20 cases reported by Gribetz in 1981 (3) and a report by one of the present institutions presented in 2016 (6). In the study of Gribetz, 12 (60\%) of those cases were caused by MAC, as isolated nodules form easily as compared to patients with an $M$. tuberculosis infection. In the present study, only two institutions (Osaka University, Osaka Toneyama Medical Center) noted cases of tuberculoma 
Table 4 Details of worsening case

\begin{tabular}{|c|c|c|c|c|c|c|c|c|c|c|c|c|c|}
\hline Case & Age & Sex & Numbers & Pathogen & $\begin{array}{l}\text { Size } \\
(\mathrm{mm})\end{array}$ & $\begin{array}{c}\text { Primary } \\
\text { lobe }\end{array}$ & Procedure & Approach & $\begin{array}{c}\text { Postoperative } \\
\text { therapy } \\
\text { (month) }\end{array}$ & $\begin{array}{l}\text { Period } \\
\text { after } \\
\text { surgery } \\
\text { (month) }\end{array}$ & \multicolumn{3}{|c|}{ At worsening } \\
\hline 1 & 64 & M & Single & MAC & 10 & LLL & Wedge & VATS & 0 & 1 & + & + & RUL \\
\hline 2 & 80 & M & Single & MAC & 10 & RUL & Wedge & VATS & 0 & 29 & + & + & RLL \\
\hline 4 & 54 & M & Single & MAC & 18 & RLL & Wedge & VATS & 0 & 42 & + & - & - \\
\hline 5 & 67 & $\mathrm{~F}$ & Single & MAC & 14 & RUL & Wedge & VATS & 0 & 24 & + & - & - \\
\hline 6 & 58 & $M$ & Single & MAC & 15 & LLL & Wedge & Open & $\mathrm{C}$ & 60 & - & + & RLL \\
\hline 10 & 75 & $\mathrm{~F}$ & Single & MAC & 13 & RML & Lobectomy & VATS & 0 & 62 & - & + & LUL \\
\hline
\end{tabular}

C, clarithromycin; E, ethambutol; L, levofroxasin; LLL, light lower lobe; LUL, left upper lobe; MAC, mycobacterium avium complex; R, rifampicin; RLL; right lower lobe; RUL, right upper lobe; VATS, video assisted thoracic surgery; RML, right middle lobe.

during the same period [2 of $22(9.1 \%)$ and 20 of $50(40 \%)$, respectively], thus it is considered that isolated nodule formation is more likely to occur with NTM as compared to $M$. tuberculosis infection. MAC was the most common species in the present SN-NTM cases, the same as noted in previous reports $(3,5-9)$, while nodules due to $M$. kansasii were noted in 8 . Huang also reported SN-NTM caused by M. kansasii in $19 \%$ of their cases, followed by MAC (10). Other reports have noted nodule formation caused by $M$. gordonae (11), M. fortuitum (12), and M. abscessus (13), while this is the first known report that includes a case caused by M. peregrinum.

It is not clear why isolated nodules are formed in cases of SN-NTM type. Solitary pulmonary nodules due to tuberculosis, termed tuberculoma, appear as an epithelial granuloma. In addition to tuberculoma, phagocytosis and accumulation of macrophages with delayed hypersensitivity reactions cause granuloma formation in cases with MAC infection (14). None of the present cases showed FC/NB type reinfection, thus individual host immune response might differ between SN-NTM and FC/NB types.

It is considered that distinction between lung cancer and SN-NTM with CT imaging is difficult, because a pulmonary nodule due to NTM often shows spiculation or indentation, similar to lung cancer. Furthermore, it is not uncommon for SN-NTM type to show a moderate accumulation of FDG in FDG-PET findings, making preoperative diagnosis more difficult using imaging results $(14,15)$. FDG accumulates in granulation species formed by activation of macrophages in cases of MAC infection, the most common species in SN-NTM type cases, and the positive rate shown by FDG-PET findings is high because the most common species is MAC, though a previous study noted that $M$. kansasii also showed a high level of accumulation (16). In the present series, FDG-PET showed accumulation of FDG in cases other than MAC infection, including 2 of 5 M. kansasii and 1 of 2 M. gordonae infection cases. Also, an $M$. peregrinum infection case showed strong accumulation with an SUV max value of 13.2. When disease activity is high in a patient affected by mycobacterial disease, stronger accumulation will be seen (17), thus diagnosis of benign or malignancy based on FDG-PET imaging results is considered to be difficult. In clinical practice, SN-NTM type combined with lung cancer has been reported (18).

The accuracy of diagnosis based on bronchoscopy biopsy results varies depending on the report, with that shown in $8.3 \%, 11.1 \%, 33.3 \%$, and $75 \%$, respectively, of previously reported cases $(3,6-8)$, while only $7.4 \%$ of the present cases received such a diagnosis. Since most cases of SN-NTM type with MAC infection have been reported to occur in peripheral areas near the pleura (9), a CT-guided biopsy would have been more effective in the present patients, 
Table 5 Comparisons between no relapse and worsening cases

\begin{tabular}{|c|c|c|c|}
\hline Variables & No relapse & Worsening & $P$ value \\
\hline Sex & & & 1.000 \\
\hline Female & 35 & 4 & \\
\hline Male & 43 & 6 & \\
\hline Age & & & 0.225 \\
\hline$<60$ years & 31 & 2 & \\
\hline$\geq 60$ years & 47 & 8 & \\
\hline Numbers & & & 0.607 \\
\hline Single & 69 & 8 & \\
\hline Multiple & 9 & 2 & \\
\hline Symptom & & & 0.531 \\
\hline None & 72 & 9 & \\
\hline Presence & 5 & 1 & \\
\hline Operation & & & 0.163 \\
\hline Wedge & 68 & 7 & \\
\hline Not wedge & 10 & 3 & \\
\hline Approach & & & 0.697 \\
\hline VATS & 60 & 7 & \\
\hline Open & 18 & 3 & \\
\hline Complications & & & 1.000 \\
\hline No & 72 & 10 & \\
\hline Yes & 6 & 0 & \\
\hline \multicolumn{2}{|c|}{ Postoperative chemotherapy } & & 0.226 \\
\hline No & 62 & 6 & \\
\hline Yes & 16 & 4 & \\
\hline
\end{tabular}

though few such examinations were performed.

According to the ATS guidelines, postoperative chemotherapy is not required for MAC infected cases when a complete surgical resection is performed, while surgical resection is not the first choice of treatment for SN-NTM type cases. A natural fading case has also been reported (19), thus differences among host defense systems are possible, including cellular immunity (3), though it is unknown whether SN-NTM type can develop into NTMPD type following resection or chemotherapy. Analysis of non-resected SN-NTM cases would provide important information.

When peripheral lesions are found near the pleura, the most common site of occurrence in SN-NTM type, a wedge resection using a thoracoscopic surgery procedure can be easily performed. As for the resection amount, in NTM-PD of FB/NB type cases, an anatomical resection has been recommended and postoperative results were also good (4,20-22). On the other hand, our previous report is the only one that includes discussion regarding the surgical procedure with respect to SN-NTM type (6). In that study, we noted that a wedge resection would be sufficient not only for purely isolated nodules, but also for cases with small cavity formation and those where the scatter nest remains in the near vicinity of the nodule, because there were no complications related to local infection, such as pneumonia or empyema, or local recurrence in any of those wedge resection cases. Furthermore, most had no relapse or reinfection. In the present series, there were also no perioperative complications noted in the wedge resection cases and the 2 cases of relapse in the partial resected lobe were not local recurrence around the cutting line. Thus, a wedge resection is considered adequate and anatomical resection is not required if there are no remaining obvious lesions around the cutting end. Although there were no cases of local recurrence, some showed reinfection after a long period following the resection. We speculated that environmental and/or immune factors were causative in those cases. However, it was difficult to identify factors related to risk of reinfection because the observation periods were not consistent in this study. Since SN-NTM is a benign disease, long-term follow-up examinations may not be necessary, though affected patients should be informed of the possibility of later reinfection.

Postoperative chemotherapy is unnecessary in cases of completely excised MAC SN-NTM type (1), while recurrence was not observed even in previously reported cases without additional chemotherapy $(6,7,11)$. On the other hand, an anti-tuberculous agent is effective against the M. kansasii species, as affected patients often present a solitary nodule that can be resected when a diagnosis is not obtained. Huang et al. reported no postoperative relapse in 8 M. kansasii cases that underwent resection after only a single postoperative treatment, which was similar to the present findings, as no relapse was observed in any of the 8 cases, including 7 in which postoperative treatment was not administered. It is considered that SN-NTM type caused by $M$. kansasii likely does not require postoperative chemotherapy after resection, the same as with MAC.

The present study is limited by its retrospective nature and inclusion of only patients who underwent excision. In 
addition, several of the cases had a short observation period, thus there is a high possibility that not all instances of reinfection were analyzed, resulting in low rates pf detection of reinfection and recurrence. On the other hand, this report includes a greater number of cases than previously reported and relevant clinical background findings were clarified by multi-center participation. It is unknown whether SN-NTM type is significantly affected by chemotherapy treatment alone or no treatment, because of the few cases reported. In most affected patients, it is difficult to distinguish from lung cancer, though a peripheral nodule limited resection procedure is available and postoperative chemotherapy may not be necessary. Nevertheless, the possibility of reinfection after resection should be explained to the patient. Since the number of analyzed cases in the present report is few, it is necessary to accumulate SN-NTM type results, including non-resected cases.

\section{Conclusions}

SN-NTM type occurring in the vicinity of the pleura is most often caused by MAC infection, though it is difficult to diagnose based on preoperative examination results or distinguish from lung cancer. We found no local complications or recurrence in any of the present analyzed cases, even those that underwent a wedge resection, thus postoperative chemotherapy is not considered necessary if completely resected.

\section{Acknowledgments}

Funding: None.

\section{Footnote}

Reporting Checklist: The authors have completed the STROBE reporting checklist. Available at http://dx.doi. org/10.21037/jtd-20-3108

Data Sharing Statement: Available at http://dx.doi. org/10.21037/jtd-20-3108

Conflicts of Interest: All authors have completed the ICMJE uniform disclosure form (available at http://dx.doi. org/10.21037/jtd-20-3108). The authors have no conflicts of interest to declare.

Ethical Statement: The authors are accountable for all aspects of the work in ensuring that questions related to the accuracy or integrity of any part of the work are appropriately investigated and resolved. The study was conducted in accordance with the Declaration of Helsinki (as revised in 2013). This study was initially approved by the Institutional Review Board of Osaka University Hospital (project approval No.17270) in December 2017 and thereafter by each of the participating institutions. Individual consent for this retrospective analysis was waived.

Open Access Statement: This is an Open Access article distributed in accordance with the Creative Commons Attribution-NonCommercial-NoDerivs 4.0 International License (CC BY-NC-ND 4.0), which permits the noncommercial replication and distribution of the article with the strict proviso that no changes or edits are made and the original work is properly cited (including links to both the formal publication through the relevant DOI and the license). See: https://creativecommons.org/licenses/by-nc-nd/4.0/.

\section{References}

1. Griffith DE, Aksamit T, Brown-Elliott BA, et al. ATS Mycobacterial Diseases Subcommittee; American Thoracic Society; Infectious Disease Society of America. An official ATS/IDSA statement: diagnosis, treatment, and prevention of nontuberculous mycobacterial diseases. Am J Respir Crit Care Med 2007;175:367-416.

2. Haworth CS, Banks J, Capstick T, et al. British Thoracic Society guidelines for the management of non-tuberculous mycobacterial pulmonary disease (NTM-PD). Thorax 2017;72:ii1-64.

3. Gribetz AR, Damsker B, Bottone EJ, et al. Solitary pulmonary nodules due to nontuberculous mycobacterial infection. Am J Med 1981;70:39-43.

4. Mitchell JD, Bishop A, Cafaro A, et al. Anatomic lung resection for nontuberculous mycobacterial disease. Ann Thorac Surg 2008;85:1887-92.

5. von Elm E, Altman DG, Egger M, et al. The Strengthening the Reporting of Observational Studies in Epidemiology (STROBE) Statement: Guidelines for Reporting Observational Studies. PLoS Med 2007;4:e296.

6. Ose N, Maeda H, Takeuchi Y, et al. Solitary pulmonary nodules due to non-tuberculous mycobacteriosis among 28 resected cases. Int J Tuberc Lung Dis 2016;20:1125-9.

7. Lim J, Lyu J, Choi CM, et al. Non-tuberculous mycobacterial diseases presenting as solitary pulmonary nodules. Int J Tuberc Lung Dis 2010;14:1635-40. 
8. Hahm CR, Park HY, Jeon K, et al. Solitary pulmonary nodules caused by Mycobacterium tuberculosis and Mycobacterium avium complex. Lung 2010;188:25-31.

9. Yonemori K, Tateishi U, Tsuta K, et al. Solitary pulmonary granuloma caused by Mycobacterium avium-intracellulare complex. Int J Tuberc Lung Dis 2007;11:215-21.

10. Huang HL, Liu CJ, Lee MR, et al. Surgical resection is sufficient for incidentally discovered solitary pulmonary nodule caused by nontuberculous mycobacteria in asymptomatic patients. PLoS One 2019;14:e0222425.

11. Collop NA. A solitary pulmonary nodule due to Mycobacterium gordonae. Respiration 1990;57:351-2.

12. Pesce RR, Fejka S, Colodny SM. Mycobacterium fortuitum presenting as an asymptomatic enlarging pulmonary nodule. Am J Med 1991;91:310-2.

13. Kwon YS, Koh WJ, Kwon OJ, et al. Mycobacterium abscessus pulmonary infection presenting as a solitary pulmonary nodule. Intern Med 2006;45:169-71.

14. Bandoh S, Fujita J, Ueda Y, et al. Uptake of fluorine-18fluorodeoxyglucose in pulmonary Mycobacterium avium complex infection. Intern Med 2003;42:726-9.

15. Kawate E, Yamazaki M, Kohno T, et al. Two cases with solitary pulmonary nodule due to nontuberculous mycobacterial infection showing intense $18 \mathrm{~F}$-fluorodeoxyglucose uptake on positron emission tomography scan. Geriatr Gerontol Int 2010;10:251-4.

16. Abe M, Kobashi Y, Mouri K, et al. Solitary pulmonary

Cite this article as: Ose N, Takeuchi Y, Kitahara N, Matumura A, Kodama K, Shiono H, Susaki Y, Funakoshi Y, Takabatake H, Shintani Y; for the Thoracic Surgery Study Group of Osaka University (TSSGO). Analysis of pulmonary nodules caused by nontuberculous mycobacteriosis in 101 resected cases: multicenter retrospective study. J Thorac Dis 2021;13(2):977-985. doi: $10.21037 /$ jtd-20-3108 nodule due to Mycobacterium kansasii. Intern Med 2011;50:775-8.

17. Demura Y, Tsuchida T, Uesaka D, et al. Usefulness of 18F-fluorodeoxyglucose positron emission tomography for diagnosing disease activity and monitoring therapeutic response in patients with pulmonary mycobacteriosis. Eur J Nucl Med Mol Imaging 2009;36:632-9.

18. Taira N, Kawasaki H, Takahara S, et al. The Presence of Coexisting Lung Cancer and Non-Tuberculous Mycobacterium in a Solitary Mass. Am J Case Rep 2018;19:748-51.

19. Nakahara Y, Mochizuki Y, Kawamura T, et al. Study on pulmonary lesions in which nontuberculous mycobacteria were detected by percutaneous aspiration--a proposal to add "culture positivity of percutaneous aspiration material" to the bacteriological diagnostic criteria of pulmonary nontuberculous mycobacterial diseases. Kekkaku 2013;88:283-9.

20. Pomerantz M, Madsen L, Goble M, et al. Surgical management of resistant mycobacterial tuberculosis and other mycobacterial pulmonary infections. Ann Thorac Surg 1991;52:1108-11.

21. Nelson KG, Griffith DE, Brown BA, et al. Results of operation in Mycobacterium avium-intracellulare lung disease. Ann Thorac Surg 1998;66:325-30.

22. Shiraishi Y, Katsuragi N, Kita H, et al. Adjuvant surgical treatment of nontuberculous mycobacterial lung disease. Ann Thorac Surg 2013;96:287-91. 